\title{
A case of longstanding primary sclerosing cholangitis
}

\author{
BM GOUDIE, GG BIRNIE, G WATKINSON, RNM MACSWEEN
}

From the Gartnavel General Hospital, and the University Department of Pathology, Western Infirmary, Glasgow

SUMMARY A 45-year-old man is described in whom there is currently ERCP and histological evidence of primary sclerosing cholangitis (PSC). A liver biopsy obtained 29 years ago shows similar histological features confirming that he had PSC at that time. This case indicates that PSC may follow a relatively benign course.

\section{Case report}

In 1952 this patient presented at the age of $16 \mathrm{yr}$ with a two-year history of diarrhoea and cramping lower abdominal pain. He was found to be clinically anaemic, physically and sexually underdeveloped and to have liver enlargement. Investigation confirmed an iron deficiency anaemia due to occult gastrointestinal blood loss but barium enema and sigmoidoscopy were normal. Associated liver disease was suggested by an increase of the alkaline phosphate to $28 \mathrm{King}$ Armstrong Units (KAU)normal range $\leqslant 25 \mathrm{KAU}$ in this age group. A year later his general condition had not improved, the liver was still enlarged and the alkaline phosphatase was still raised (30 KAU). Diagnostic laparotomy was undertaken and revealed an enlarged scarred liver. The extra-hepatic biliary tree was found to be completely normal with no evidence of gallstones. No operative procedure was attempted on the common bile duct and cholangiography was not performed. A wedge biopsy of liver was taken. This showed some portal fibrosis and a few attenuated portal-portal fibrous septa. There was a light chronic inflammatory cell infiltrate in the portal tracts (Fig. 1); the significant observation was a definite paucity of small bile ducts, small histiocytic aggregates being present at their previous site (Fig. 1a); some mild focal periportal inflammation was noted in some areas (Fig. 1b) and there were small periportal accumulations of copper-associated protein demonstrable on the orcein stain.

Three years later he developed severe diarrhoea and rectal bleeding. Sigmoidoscopy and barium enema showed typical features of ulcerative colitis. Despite an initial improvement with steroid therapy his condition deteriorated, and necessitated elective colectomy with ileorectal anastomosis in April 1957.

Accepted for publication 21 June 1983
The alkaline phosphatase was $56 \mathrm{KAU}$ at this time. Some months later he developed paralytic poliomyelitis which produced a residual paraplegia. For the next eight years he remained generally well-adapting to his neurological deficit and maturing normally. He trained as a civil servant and works to the present day. In 1965 he had a recurrence of diarrhoea and underwent anterior resection of the rectum. Histological examination confirmed continuing active ulcerative colitis.

He remained well until 1981 when he developed painless jaundice with the passage of pale stools and. dark urine. He had no fever. On clinical examinae tion there was hepatosplenomegaly but no stigmata of chronic liver disease. The liver function tests were abnormal: bilirubin $50 \mu \mathrm{mol} / 1$, alkaline phosphatase $169 \mathrm{IU} / \mathrm{l}$ (normal range 35-130 IU/l). This episode $\triangle$ of jaundice resolved spontaneously but he continued to have raised alkaline phosphatase (360 IU/l) and transaminase activities (serum AST $76 \mathrm{U} / \mathrm{l}$, serum ALT $149 \mathrm{U} / \mathrm{l}$ ). The serum albumin remained normal. Serological testing for antimitochondrial antibody was negative, the IgM level was normal and there was no eosinophilia.

An ERCP (Fig. 2) demonstrated a stricture in the 8 common bile duct with widespread stricturing and beading of the intra-hepatic biliary tree-features $\mathrm{O}$ which are characteristic of sclerosing cholangitis. ${ }^{1} \mathrm{~A}$ percutaneous liver biopsy showed more marked portal tract fibrosis with fibrous septum formation but a diagnosis of cirrhosis could not be made with cer- or tainty. In the majority of portal tracts no bile ducts $N$ could be identified; there was well marked peripor- N tal hepatitis with ballooning of liver cells, occasional $O$ Mallory bodies and heavy deposits of copper associated protein were demonstrated on the orcein stain (Fig. 3). The liver copper content was estimated by neutron activation analysis and found to be 18 times 0 normal at 440 ppm (dryweight). 


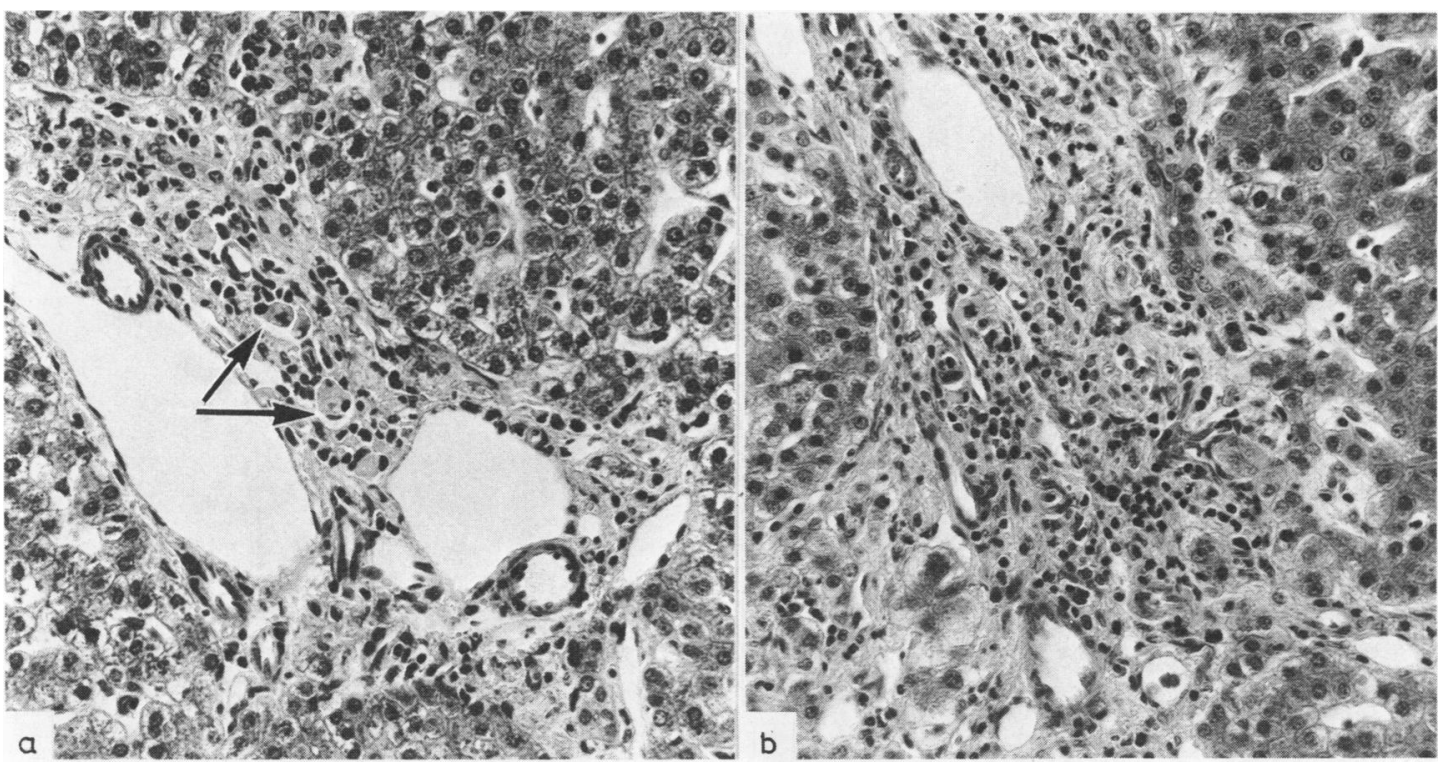

Fig. 1 Wedge biopsy of liver in 1953. (a) In this portal area there is a light chronic inflammatory cell infiltrate; no bile duct is present and an aggregate of histiocytes (arrows) marks its former site. Masson's trichrome $\times 330(b)$ In this portal area note again the absence of a bile duct; fibrous extension and some periportal hepatitis is noted in the lower half of the field. Masson's trichrome $\times 300$.

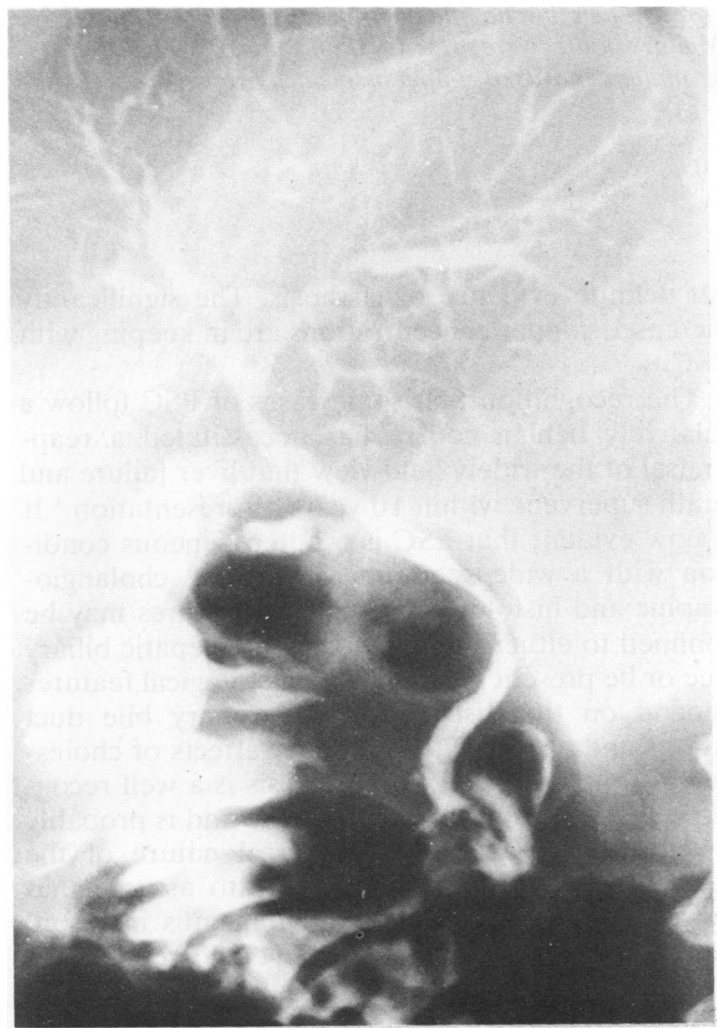

Fig. 2 ERCP in 1982. There is a common bile duct stricture and multiple intrahepatic strictures with "beading" of the ducts between narrowed segments. 

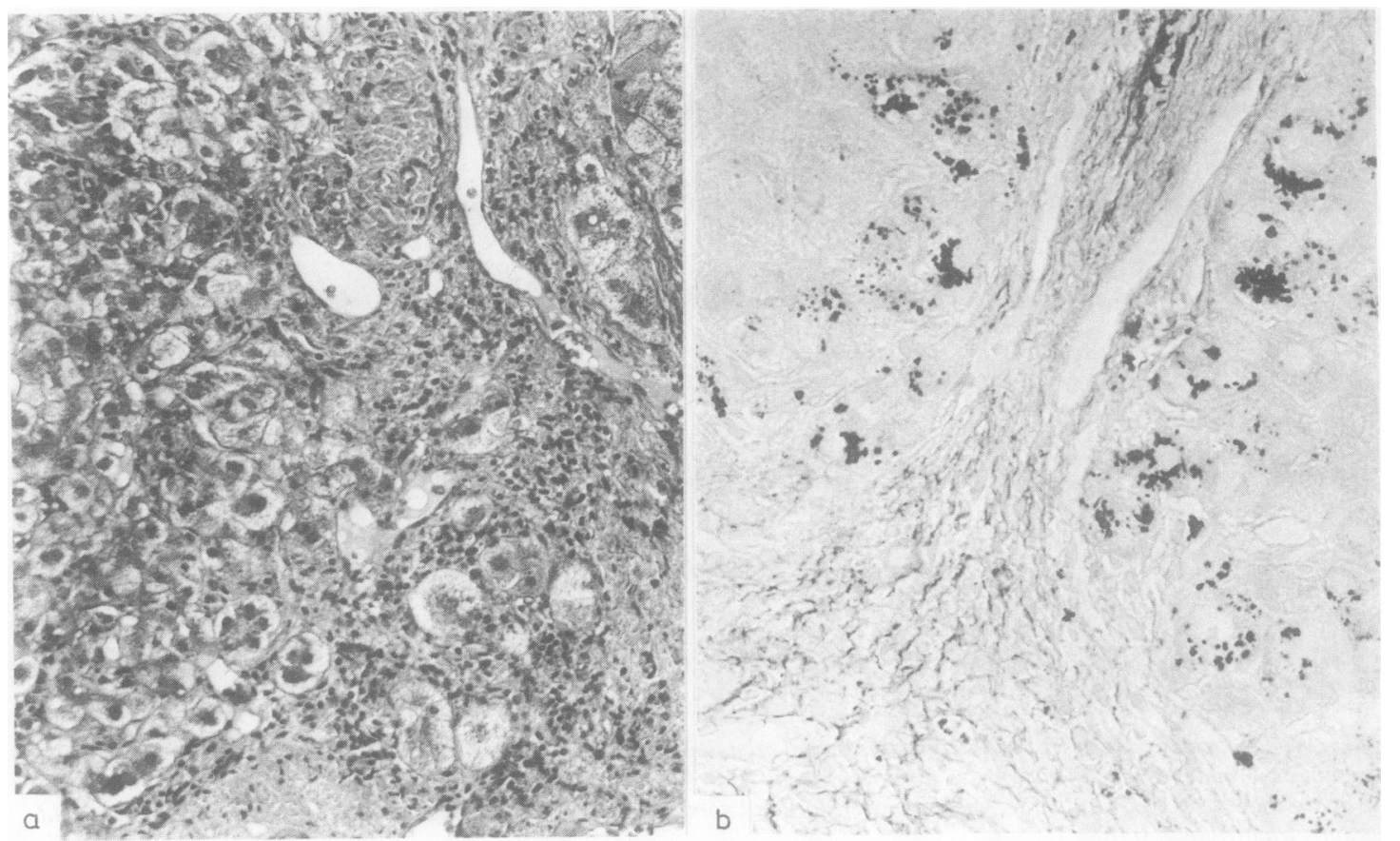

Fig. 3 Needle biopsy of liver in 1981. (a) Two portal areas are present here but no bile ducts are identifiable. There is portal-portal fibrous linkage, periportal hepatitis and occasional Mallory bodies were present within some of the ballooned liver cells. Haematoxylin and eosin $\times \mathbf{2 8 0}($ b) Well marked black granular deposits of copper associated protein are seen in periportal and periseptal hepatocytes. Shikata's orcein $\times 500$.

\section{Discussion}

PSC was first described in $1942^{2}$ and for the next 50 years regarded as an extremely rare cause of obstructive jaundice. Since 1974 when ERCP was recognised as a useful diagnostic technique ${ }^{1}$ PSC has been diagnosed with increasing frequency and asymptomatic cases are now being identified in whom the only abnormality is an increase of the hepatic alkaline phosphatase. Chapman has described prolonged survival in asymptomatic precirrhotic cases-the longest recorded survival being 15 years. ${ }^{3}$ Our patient had histological evidence of PSC at the age of $16 \mathrm{yr}$ and is still alive 30 years later. Apart from vague abdominal discomfort he had had no symptoms attributable to liver disease until a transient self-limiting episode of jaundice at the age of $44 \mathrm{yr}$. He now has hepatosplenomegaly, persistent raised serum alkaline phosphatase and transaminase activities and ERCP findings consistent with the diagnosis of PSC. His liver biopsy indicates progression of the disease although there is not yet definite evidence of cirrhosis. The significantly increased copper concentrations are in keeping with PSC. ${ }^{4}$

The recognition that some cases of PSC follow a relatively benign course has necessitated a reappraisal of the widely held view that liver failure and death supervene within 10 years of presentation. ${ }^{5}$ It is now evident that PSC is a heterogeneous condition with a wide spectrum of clinical, cholangiographic and histological severity. Strictures may be confined to either the extra- or intra-hepatic biliary tree or be present in both. ${ }^{6}$ The histological features depend on the distribution of primary bile duct lesions and also on the secondary effects of cholestasis. ${ }^{5}$ Slow progression to cirrhosis is a well recognised feature of biliary tract disease and is probably a reflection of the segmental/focal nature of the early lesions. It seems reasonable to assume that those patients with sclerosing cholangitis in whom there is only focal intrahepatic duct involvement will have minimal cholestasis and as a consequence only slowly progressive disease. On the other hand, 
patients in whom there is marked cholestasis, as the result of widespread intrahepatic or major duct strictures, would be expected to sustain more severe hepatocellular damage and to have a poorer prognosis. The activity of an associated colitis does not appear to be related to the progression of PSC and colectomy does not confer protection against its development. $^{4}$ At the present time there is insufficient information about the aetiology and natural history of the disease to permit prediction of prognosis in individual patients.

The inability to anticipate the course of disease in asymptomatic cases has important implications where the use of potentially toxic drugs is being considered. Clearly any therapeutic trial must be conducted on a carefully controlled basis.

We wish to thank Mr Gordon Gillespie for performing the ERCP.

\section{References}

' Elias E, Summerfield JA, Dick K, Sherlock S. Endoscopic retrograde cholangio-pancreatography in the diagnosis of jaundice associated with ulcerative colitis. Gastroenterology 1974;67:907-11.

${ }^{2}$ Delbet P. Retrecissement du choledoque: Cholecystoduodenostomie. Bull Mem Soc Nation Chirurg 1924;50:1144-6.

${ }^{3}$ Chapman RWG, Burroughs AK, Bass NM, Sherlock S. Longstanding asymptomatic primary sclerosing cholangitis: Report of three cases. Dig Dis Sci 1981;26:778-82.

4 Chapman RWG, Arborgh BAM, Rhodes JM, et al. Primary sclerosing cholangitis: A review of its features, cholangiography and hepatic histology. Gut 1980;21:870-7.

5 Lefkowitch JH. Primary sclerosing cholangitis. Arch Intern Med 1982;142:1157-60.

6 Wiesner RH, La Russo NF. Clinicopathologic features of the syndrome of primary sclerosing cholangitis. Gastroenterology 1980;79:200-6.

Requests for reprints to: Professor RNM MacSween, University Department of Pathology, Western Infirmary, Glasgow G11 6NT, Scotland. 